\title{
Apparent brightness distribution of GRB host galaxies
}

\author{
Zsolt Bagoly $^{1}$, István I. Rácz ${ }^{1,2}$, Lajos G. Balázs ${ }^{1,2}$, István Horváth ${ }^{3}$, \\ and L. Viktor Tóth ${ }^{1}$ \\ ${ }^{1}$ Eötvös University, Budapest, email: zsolt.bagoly@ttk.elte.hu \\ ${ }^{2}$ Konkoly Observatory of the Hungarian Academy of Sciences, Budapest, ${ }^{3}$ National University \\ of Public Service, Budapest
}

\begin{abstract}
We studied the unbiased optical brightness distribution which was calculated from the survival analysis of host galaxies (HGs) data and its relationship with the Swift GRB data of the host galaxies observed by the Keck telescope. Based on the sample obtained from merging the Swift GRB table and the Keck optical data we also studied the dependence of this distribution on the GRB's data. Finally, we compared the HGs distribution with standard galaxies distribution of the DEEP2 redshift survey and checked the result with the VIPERS catalogue too.
\end{abstract}

Keywords. methods: analytical, methods: data analysis, methods: statistical, catalogs, surveys, Galaxy: fundamental parameters, gamma rays: bursts, galaxies: photometry

\section{Introduction}

Most LGRBs are identified with massive stellar explosions, frequently in star-forming galaxies. Many host galaxies (HGs) are observed and it is an open question yet whether the luminosity distribution of HGs, characterized by GRB events, is identical with those of the galaxies in general. Unfortunately, many HGs are fainter than the detection limit of optical observations. Nevertheless, one can give an upper limit of their luminosity (Savaglio et al. (2009)). Using this extra information we apply the survival analysis (e.g. Feigelson \& Nelson (1985), Isobe et al. (1986), Diez et al. (2013)) to help determine the unbiased estimate of the luminosity distribution.

\section{Results}

For searching host galaxies more than 160 GRB positions were observed in the Preliminary Keck GRB Host Project (Perley et al. (2013)). Since the GRBs are connected to the standard candle luminosity function assumption, we compared the HGs luminosity distribution with DEEP2 Galaxy Redshift Survey (Newman et al. (2013)). Galaxies are sampled between about 21 and 24 magnitude on both colors so we can find significant difference in two cases, the probabilities of random differences are only $3 \cdot 10^{-5}$ in I band and $9 \cdot 10^{-5}$ in $\mathrm{R}$ band. The results are shown in Fig. 1.: the probability is three times smaller in I color, which shows that GRB host galaxies are bluer than normal galaxies.

Moreover we compared the two independent galaxy samples, the DEEP2 and VIMOS Public Extragalactic Redshift Survey (VIPERS) (Guzzo et al. (2014), Garilli et al. (2014), Jenster et al. (2005)). We found significant difference between the DEEP2 and VIPERS photometry data in $\mathrm{R}$ and $\mathrm{I}$ bands. 

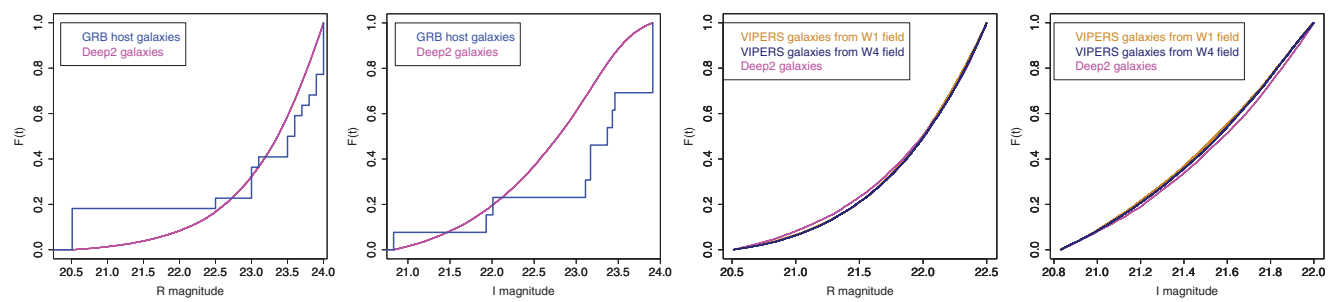

Figure 1. The comparison of the distribution function obtained from the survival function I color and the DEEP2 I color distribution (left) and $\mathrm{R}$ color (middle left). The brightness distribution of the hosts are systematically fainter than the DEEP2 galaxies, the probability of random difference is $9 \cdot 10^{-5}$ in $\mathrm{R}$ band and only $3 \cdot 10^{-5}$ in I band. At the right side we show the significant difference between the DEEP2 and VIPERS (in all fields) photometry data $\left(\rho \leqq 2 \cdot 10^{-16}\right)$ in $\mathrm{R}$ and I bands respectively.

\section{Summary and conclusions}

Comparing the cumulative distribution functions of the survival analysis of the GRB hosts with the DEEP2 sample in the $\mathrm{R}$ and $\mathrm{I}$ bands we recognized an offset of hosts towards fainter magnitudes. This offset is significant at the $9 \cdot 10^{-5}$ level in the $\mathrm{R}$ and $3 \cdot 10^{-5}$ in the I band. Assuming the same spatial distribution of the GRB hosts and the DEEP2 sample galaxies this offset is explained by the lower absolute brightness and probably systematically lower mass of the GRB hosts. The offset in the R band seems to be smaller than in the I band indicating a bluer R-I color index of the GRB hosts. The bluer color index may indicate a higher star forming activity in the hosts. On the other hand, the VIPERS galaxy sample (on $24 \mathrm{deg}^{2}$ ) and DEEP2 (on $2.8 \mathrm{deg}^{2}$ ) shows significant difference in brightness distribution based on a Kolmogorov-Smirnov Test.

The dependence of the GRB host galaxies brightness distribution on other GRB parameters (BAT T90, Fluence, 1-sec Peak Photon Flux, Photon Index, XRT 11 Hour Flux, XRT 24 Hour Flux and redshift) show no correlation.

Finally, for the investigated GRBs no correlation were found among the type of the best fitted shape of the spectra (PL or $\mathrm{CPL}$ ) and whether their redshift are known or not.

\section{Acknowledgments}

This work was supported by the Hungarran OTKA NN-111016 grant. Funding for the DEEP2 Galaxy Redshift Survey has been provided by NSF grants AST-95-09298, AST-0071048, AST0507428, and AST-0507483 as well as NASA LTSA grant NNG04GC89G. This paper uses data from the VIMOS Public Extragalactic Redshift Survey (VIPERS). VIPERS has been performed using the ESO Very Large Telescope, under the "Large Programme" 182.A-0886. The participating institutions and funding agencies are listed at http://vipers.inaf.it

\section{References}

Diez, D., Survival Analysis in R, 2013, OpenIntro.org

Feigelson, E. D. and Nelson, P. I., 1985, ApJ 2093, 192F

Garilli, B. et al., 2014, A\&A 562A, 23G

Guzzo, L. et al., 2014, A\&A 566A ,108G

Isobe, T. et al., 1986, ApJ306, 490I

Jester et al., 2005, AJ130, 873J

Newman, J. A. et al.,2013, ApJS 208:5

Perley, D. et al., 2013, EAS Publications Series Vol. 61

Savaglio, S. et al., 2009, ApJ 691, 182 